\title{
IdeaGens: Enabling Expert Facilitation of Crowd Brainstorming
}

\section{Joel Chan}

Human-Computer Interaction Institute

Carnegie Mellon University

Pittsburgh, PA, USA

joelchuc@cs.cmu.edu

\section{Steven Dang}

Human-Computer Interaction Institute

Carnegie Mellon University

Pittsburgh, PA, USA

stevenda@cs.cmu.edu

\section{Steven P. Dow}

Human-Computer Interaction Institute

Carnegie Mellon University

Pittsburgh, PA, USA

spdow@cs.cmu.edu

Permission to make digital or hard copies of part or all of this work for personal or classroom use is granted without fee provided that copies are not made or distributed for profit or commercial advantage and that

copies bear this notice and the full citation on the first page. Copyrights copies bear this notice and the full citation on the first page. Copyrights
for third-party components of this work must be honored. For all other uses, contact the Owner/Author.

Copyright is held by the owner/author(s).

CSCW '16 Companion, February 27 - March 02, 2016, San Francisco, CA USA

ACM 978-1-4503-3950-6/16/02

http://dx.doi.org/10.1145/2818052.2874313

\begin{abstract}
Online crowds are a promising source of new innovations. However, crowd innovation quality does not always match its quantity. One way to improve quality is to enable experts to provide personalized feedback. However, this scales poorly, and may lead to premature convergence during creative work. To deal with these issues, we present IdeaGens, a crowd ideation system that adapts expert facilitation, a successful strategy for improving collaborative creativity in face-to-face brainstorms, to crowd brainstorming. In IdeaGens, experts monitor incoming ideas from the crowd through a dashboard, and offer high-level "inspirations" to guide ideation towards interesting solution themes. In a randomized controlled experiment, crowd workers who receive facilitation through IdeaGens generate significantly more creative ideas that unfacilitated crowd workers.
\end{abstract}

\section{Author Keywords}

Creativity; crowdsourcing; expert facilitation

\section{ACM Classification Keywords}

H.5.3. Information interfaces and presentation (e.g., HCI): Group and Organization Interfaces: Computersupported cooperative work. 


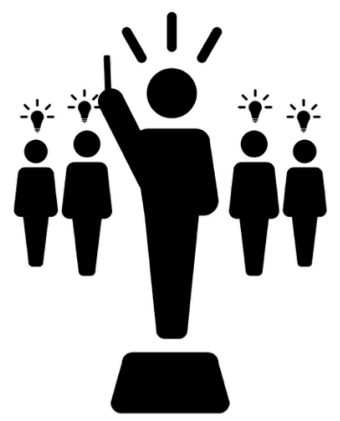

Figure 1: Expert input can significantly improve crowd work, but can be challenging to implement for complex creative crowd work. IdeaGens is designed to meet this challenge.

\section{Introduction}

Organizations increasingly turn to online crowds to obtain fresh perspectives on challenging problems. Theoretically, the scale and diversity of crowds offer increased chances of obtaining exceptional solutions. In practice, however, crowds excel at generating many ideas, but often fail to reliably generate many creative (i.e., both novel and valuable) ideas. For example, Dell's IdeaStorm platform has implemented 550 product ideas gathered from the crowd, but these are laboriously culled from more than twenty thousand idea submissions, many of which are duplicate ideas or too vague/impractical to add value as a new product.

Prior research has explored strategies for integrating experts into crowd innovation processes, e.g., establishing creative goals [4], or providing timely, task-specific feedback [2]. These strategies improve creative outcomes, but can be difficult to perform at crowd scale. Further, while expert guidance can help crowds solutions, it might hinder divergent thinking. For example, showing workers exemplary solutions could lead to premature convergence during creative tasks, since people often have a hard time breaking away from past successful solutions $[1,7]$.

One successful strategy for simultaneously improving divergence and convergence (in face-to-face group brainstorming) is to employ a skilled facilitator $[3,10]$, who improves the group's ideation by providing inspiring images or prototypes [8] and calling attention to emergent themes and unique ideas [10]. For example, a common facilitation strategy is to say, " $X$ is an interesting idea. How else might we <leverage feature $Y$ of idea $X>$ ?" Prior studies show that face-toface groups with a dedicated facilitator outperform groups with no facilitation in terms of both divergent and convergent performance $[3,5]$. IdeaGens aims to adapt this face-to-face strategy for use in crowd brainstorming, where many tens to hundreds of people (vs. 6-10 people) are ideating simultaneously.

\section{Technical Overview}

IdeaGens is designed according to the following design guidelines:

- Responsiveness: Enable facilitators to monitor and responsively guide ideation as it unfolds over time

- Flexibility: Support a range of inspiration strategies that apply to diverse types of innovation problems

- Scalability: Allow one or a few people to manage a large crowd of workers

IdeaGens is built in MeteorJS, a full-stack Javascript web application framework built on Node.js. The system includes an ideator interface where crowd workers can generate ideas in parallel, and a facilitation dashboard that enables real-time monitoring and guiding of the crowd's ideation. The core of IdeaGens is an inspiration system that links the dashboard and individual ideator interfaces. The dashboard enables facilitators to create inspirations (as open-ended textbased messages) that call out interesting themes or frame the problem in new ways (see Fig. 2).

One key design consideration is how to distribute inspirations across ideators. In typical face-to-face brainstorms, facilitators typically "push" guidance, gently interrupting the discussion at an appropriate time (e.g., during lulls in the discussion) with prompts or questions that are tailored to the group's discussion. However, we felt that this "push" model would not scale 


\begin{tabular}{|c|c|c|}
\hline Remember names & 357 Ideas by 28 Ideators 0 active & \begin{tabular}{|l} 
Create new \\
Inspiration...
\end{tabular} \\
\hline Keyword search & \multirow{6}{*}{ 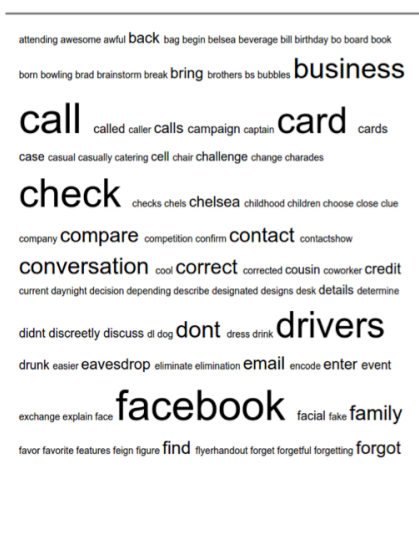 } & \\
\hline Sort: Most Recent $/$ & & $\begin{array}{l}\text { Create } \\
11 \text { nspirations }\end{array}$ \\
\hline $\begin{array}{l}\text { jokingly shout } \\
\text { "Hey does } \\
\text { anyone know } \\
\text { this person?" }\end{array}$ & & $\begin{array}{l}\text { What might } \\
\text { you do if you } \\
\text { weren't easily } \\
\text { embarassed? }\end{array}$ \\
\hline $\begin{array}{l}\text { Start to sing } \\
\text { Happy Birthday } \\
\text { and get others } \\
\text { to join in that } \\
\text { know the name }\end{array}$ & & 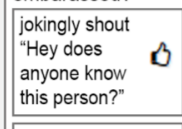 \\
\hline \begin{tabular}{|l|} 
say let me \\
guess your \\
name is george \\
washington
\end{tabular} & & $\begin{array}{l}\text { say lets all sing } \\
\text { our own verse } \\
\text { of the "name } \\
\text { game" song, I'll } \\
\text { go first }\end{array}$ \\
\hline $\begin{array}{l}\text { ask if they are } \\
\text { named after } \\
\text { any family me- }\end{array}$ & & $\begin{array}{l}\text { Peoples names } \\
\text { are often on }\end{array}$ \\
\hline
\end{tabular}

Figure 2. Dashboard enables facilitators to monitor the evolving solution space, as well as guide crowd ideation through the creation of inspirations. Facilitators also receive feedback on their inspirations by inspecting ideas that were inspired by each inspiration.

to facilitating many tens to potentially hundreds of ideators working in parallel. Indeed, in pilot testing with earlier iterations of the tool, we found that facilitators were not able to effectively and efficiently decide when and to whom to distribute inspirations, even with as few as 8-10 ideators. Therefore, we implemented a "pull" mechanism for inspiration distribution.

The system collects inspirations in a queue, which ideators can "pull" from on-demand in a simple first-infirst-out algorithm (i.e., older inspirations pulled first). At any time they wish, ideators can press the "Inspire $\mathrm{Me}^{\prime \prime}$ button (located below the brainstorming prompt) to pull new inspirations from the inspiration queue (see Fig. 3). Each button press yields a single new

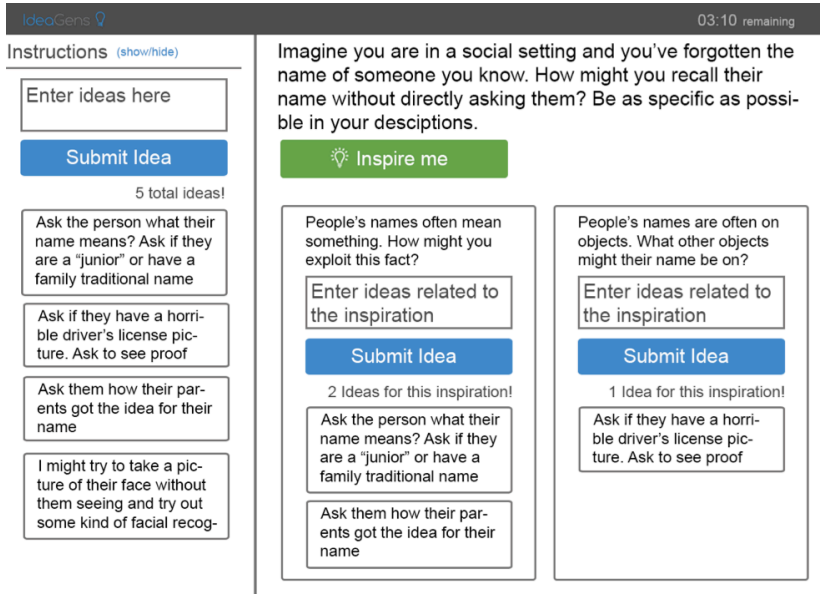

Figure 3. Ideator interface allows ideators to receive inspirations on-demand by clicking on the "Inspire $\mathrm{Me}^{\text {" button. }}$ To provide feedback to facilitators, ideators are encouraged to enter ideas sparked by an inspiration into the inspirationspecific entry box.

inspiration, which appears directly below the button. The system keeps a tally of the number of ideators and ensures that there are always enough "copies" of each inspiration for all workers to access if they choose. This "pull" approach supports greater scalability and was motivated by prior findings that ideators benefit most from inspirations when delivered "on demand" [9].

\section{Evaluation}

We evaluated IdeaGens with a controlled experiment. Crowd workers $(N=87)$ on Amazon Mechanical Turk ideated solutions for a common social predicament (forgetting an acquaintance's name). Participants either brainstormed independently with no facilitation or received high-level guidance (i.e., inspirations) from 


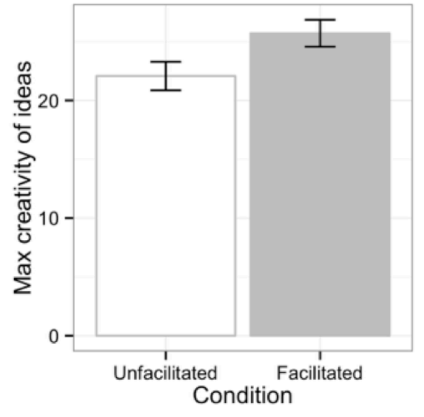

Figure 4: Crowd workers generate more creative ideas when facilitated using IdeaGens, compared to unfacilitated crowd workers. two facilitators with prior experience managing brainstorming sessions. Facilitated participants generated more ideas of higher creativity (as rated by blind-to-condition judges) than unfacilitated participants, $\mathrm{F}(1,83)=4.8, p=0.03$ (see Fig. 4).. Additionally, as measured by Latent Semantic Analysis [6], facilitated participants had higher convergence (i.e., higher occurrence of highly similar idea pairs, an index of design iteration), $\mathrm{F}(1,83)=3.2, p=0.08$, but nevertheless also had equal divergence (semantic diversity of ideas) as unfacilitated participants, $F(1,83)=0.6, p=0.45$, indicating that facilitation through IdeaGens enables crowd workers to strike a good balance between convergence and divergence.

\section{Conclusion}

To address difficulties obtaining creative ideas from the crowd, we designed and evaluated IdeaGens, a system for guided crowd brainstorming. We look forward to sharing this system with the CSCW community.

\section{References}

1. Barry L. Bayus. 2013. Crowdsourcing new product ideas over time: An analysis of Dell's Ideastorm community. Management Science 59, 1: 226-244. http://doi.org/10.1287/mnsc.1120.1599

2. Steven Dow, Anand Kulkarni, Scott Klemmer, and Björn Hartmann. 2012. Shepherding the crowd yields better work. Proceedings of the ACM 2012 conference on Computer Supported Cooperative Work, ACM, 1013-1022. http://doi.org/10.1145/2145204.2145355

3. Scott G. Isaksen and John P. Gaulin. 2005. A Reexamination of Brainstorming Research: Implications for Research and Practice. Gifted Child
Quarterly 49, 4: 315-329.

http://doi.org/10.1177/001698620504900405

4. Joy Kim, Justin Cheng, and Michael S. Bernstein. 2014. Ensemble: Exploring Complementary Strengths of Leaders and Crowds in Creative Collaboration. Proceedings of the 17th ACM Conference on Computer Supported Cooperative Work \&\# 38; Social Computing, ACM, 745-755. http://doi.org/10.1145/2531602.2531638

5. Thomas J. Kramer, Gerard P. Fleming, and Scott M. Mannis. 2001. Improving Face-To-Face

Brainstorming Through Modeling and Facilitation. Small Group Research 32, 5: 533-557. http://doi.org/10.1177/104649640103200502

6. Thomas K. Landauer, Peter W. Foltz, and Darrell Laham. 1998. An introduction to latent semantic analysis. Discourse Processes 25, 2: 259-284.

7. Abraham S. Luchins. 1942. Mechanization in problem solving: The effect of Einstellung. Psychological Monographs 54, 6: i-95. http://doi.org/10.1037/h0093502

8. Alex F. Osborn. 1963. Applied Imagination Principles and Procedures of Creative Problem Solving. Charles Scribner's Sons, New York, NY.

9. Pao Siangliulue, Joel Chan, Kzryzstof Gajos, and Steven P. Dow. 2015. Providing timely examples improves the quantity and quality of generated ideas. Proceedings of the ACM Conference on Creativity and Cognition.

10. Robert I. Sutton and A. Hargadon. 1996. Brainstorming groups in context: Effectiveness in a product design firm. Administrative Science Quarterly 41: 685-718. 